\title{
3-10-GHz Ultra-Wideband Low-Noise Amplifier Utilizing Miller Effect and Inductive Shunt-Shunt Feedback Technique
}

\author{
Yu-Tso Lin, Hsiao-Chin Chen, Tao Wang, Yo-Sheng Lin, Senior Member, IEEE, and \\ Shey-Shi Lu, Senior Member, IEEE
}

\begin{abstract}
In this paper, we demonstrate an SiGe HBT ultra-wideband (UWB) low-noise amplifier (LNA), achieved by a newly proposed methodology, which takes advantage of the Miller effect for UWB input impedance matching and the inductive shunt-shunt feedback technique for bandwidth extension by pole-zero cancellation. The SiGe UWB LNA dissipates $25.8-\mathrm{mW}$ power and achieves $S_{11}$ below $-10 \mathrm{~dB}$ for frequencies from 3 to $14 \mathrm{GHz}$ (except for a small range from 10 to $11 \mathrm{GHz}$, which is below -9 dB), flat $S_{21}$ of $24.6 \pm 1.5 \mathrm{~dB}$ for frequencies from 3 to $11.6 \mathrm{GHz}$, noise figure of 2.5 and $5.8 \mathrm{~dB}$ at 3 and $10 \mathrm{GHz}$, respectively, and good phase linearity property (group-delay variation is only \pm 28 ps across the entire band). The measured 1-dB compression point $\left(P_{1 \mathrm{~dB}}\right)$ and input third-order intermodulation point are -25.5 and $-17 \mathrm{dBm}$, respectively, at $5.4 \mathrm{GHz}$.
\end{abstract}

Index Terms-HBT, inductive feedback, low-noise amplifier (LNA), Miller effect, SiGe, ultra-wideband (UWB).

\section{INTRODUCTION}

$\mathbf{S}^{1}$ INCE THE U.S. Federal Communications Commission (FCC) approved the application of ultra-wideband (UWB) technology for commercial use in early 2002, ranging from 3.1 to $10.6 \mathrm{GHz}$ (bandwidth: $7.5 \mathrm{GHz}$ ), the implementation of a UWB system and its sub-blocks has been a challenge for integrated circuit (IC) designers. In UWB receiver design, the UWB low-noise amplifier (LNA) is a critical block of the receiver front-end. Different from that operating in a narrowband communication system, a UWB LNA has to receive small signals from 3.1 to $10.6 \mathrm{GHz}$ and amplify them with a good signal-to-noise ratio property over the entire UWB band. In addition, flat and high power gain $\left(S_{21}\right)$, input impedance matching (i.e., low input-return loss $S_{11}$ ), and good noise figure (NF) performances across the entire UWB band are also required. Recently, several UWB LNAs have been reported [1]-[11]. For some UWB LNAs [1], [2], which are designed for an orthogonal frequency division multiplexing (OFDM)

Manuscript received October 18, 2006; revised June 17, 2007. This work was supported by the National Science Council of the R.O.C. under Contract NSC95-2221-E002-364 and Contract NSC93-2752-E-002-002-PAE, and by the Ministry of Economic Affairs under Contract MOEA 94EC17A05-S1-017.

Y.-T. Lin, H.-C. Chen, T. Wang, and S.-S. Lu are with the Graduate Institute of Electronics Engineering and Department of Electrical Engineering, National Taiwan University, Taipei 10617, Taiwan, R.O.C. (e-mail: sslu@ntu.edu.tw).

Y.-S. Lin is with the Department of Electrical Engineering, National Chi Nan University, Puli 545, Taiwan, R.O.C.

Color versions of one or more of the figures in this paper are available online at http://ieeexplore.ieee.org.

Digital Object Identifier 10.1109/TMTT.2007.903836

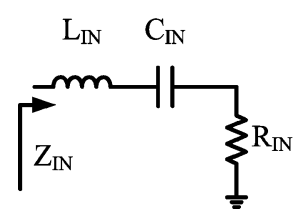

Fig. 1. RLC series network.

system, linearity is a tight requirement for suppressing adjacent channel interferences. On the other hand, for UWB pulse radio systems [3], [4], the amplitude linearity requirements are relaxed; instead, in order to keep the shape of the pulse while receiving radio signals from an antenna, good phase linearity is required.

In this paper, a new methodology to implement a UWB LNA for UWB pulse radio system applications is demonstrated by $0.35-\mu \mathrm{m}$ SiGe HBT technology. The proposed methodology takes advantage of the Miller effect for UWB input impedance matching and the inductive shunt-shunt feedback technique for bandwidth extension by pole and zero cancellation. Although inductive shunt-shunt feedback topology has been seen [12]-[14] before, their major intention is for transistor neutralization or peaking rather than circuit pole-zero cancellation, which will be detailed later in this paper. This paper is organized as follows. Section II describes the circuit design principles of UWB input impedance matching and UWB $S_{21}$. Section III introduces the proposed UWB LNA with four cascaded stages. Transfer function of $S_{21}$ of this circuit is derived for the purpose of illustrating the proposed methodology. In addition, theoretical analysis of NF and linearity is also included. Finally, the experimental results of the SiGe UWB LNA are discussed in Section IV, which is followed by conclusions in Section V.

\section{Principles of Circuit Design}

\section{A. UWB Input Impedance Matching}

To discuss UWB input impedance matching, consider the frequency response of $S_{11}$ of an $R L C$ series network shown in Fig. 1 first. The input impedance $Z_{\mathrm{IN}}$ of it is given by

$$
Z_{\mathrm{IN}}=s L_{\mathrm{IN}}+R_{\mathrm{IN}}+\frac{1}{s C_{\mathrm{IN}}}
$$


According to (1), $S_{11}$ can be expressed as follows:

$$
\begin{aligned}
S_{11} & =\frac{Z_{\mathrm{IN}}-R_{S}}{Z_{\mathrm{IN}}+R_{S}} \\
& \approx \frac{s^{2}+s \frac{R_{\mathrm{IN}}-R_{s}}{L_{\mathrm{IN}}}+\frac{1}{C_{\mathrm{IN}} L_{\mathrm{IN}}}}{s^{2}+s \frac{R_{\mathrm{IN}}+R_{s}}{L_{\mathrm{IN}}}+\frac{1}{C_{\mathrm{IN}} L_{\mathrm{IN}}}} \\
& \equiv \frac{s^{2}+\omega_{O}^{2}}{s^{2}+s \frac{\omega_{O}}{Q_{\mathrm{IN}}}+\omega_{O}^{2}}\left(\text { or } \frac{s^{2}+\omega_{O}^{2}}{s^{2}+s B+\omega_{O}^{2}}\right)
\end{aligned}
$$

in which $\omega_{0}$ is called the pole frequency (or center frequency) and $Q_{\mathrm{IN}}=\omega_{O} L_{\mathrm{IN}} /\left(R_{\mathrm{IN}}+R_{s}\right)$ is called the pole $Q$ factor [15]. Now it is clear that $\left|S_{11}\right|$ is a standard notch function with -3-dB bandwidth $\Delta \omega_{3 \mathrm{~dB}}$ equal to $B=\omega_{o} / Q_{\mathrm{IN}}$ [16]. However, in RF design, $\left|S_{11}\right|$ should be smaller than $-10 \mathrm{~dB}$ over the band of interest, i.e.,

$$
20 \log \left|S_{11}\right| \leq-10 \mathrm{~dB}
$$

By substituting the expression of $S_{11}$ in (2) into (3) and after some calculations, it was found that the input impedance matching bandwidth $\Delta f_{10 \mathrm{~dB}}$ is equal to

$$
\Delta f_{10 \mathrm{~dB}}=\frac{\Delta \omega_{10 \mathrm{~dB}}}{2 \pi}=\frac{B}{6 \pi}=\frac{\omega_{O}}{6 \pi Q_{\mathrm{IN}}} \approx \frac{100}{6 \pi L_{\mathrm{IN}}}
$$

That is, the input matching bandwidth $\Delta f_{10 \mathrm{~dB}}$ is inversely proportional to the value of $L_{\mathrm{IN}}$ (or inversely proportional to the value of $Q_{\mathrm{IN}}$ if the value of the center frequency $\omega_{o}$ is fixed). From (4), for a 3.1-10.6-GHz UWB LNA, the inductance $L_{\mathrm{IN}}$ should not be larger than $0.707 \mathrm{nH}$ (or $Q_{\mathrm{IN}}<0.25$ ). In the traditional LNA design using series- series inductive feedback, as shown in Fig. 2(a), the input resistance $R_{\mathrm{IN}}$ (usually $50 \Omega$ ) is generated by the emitter (or a source) degenerative inductor $L_{S}$ [17], and the base inductor $L_{\mathrm{GD}}$ and the emitter inductor $L_{S}\left(L_{\mathrm{GD}}+L_{s}=L_{\mathrm{IN}}\right)$ are used to resonate in the center frequency $\left(f_{o}\right)$, as shown in Fig. 2(a). Recently, several circuit topologies have been developed for UWB input impedance matching based on the $L C$ bandpass filter theory [1] [2], in which the necessary $50 \Omega$ is still mainly generated by the emitter (or source) inductor.

The capacitive feedback technique utilizing Miller effect has been used to achieve UWB input impedance matching [18], [19] instead of generating the necessary $50 \Omega\left(R_{\mathrm{IN}}\right)$ by an emitter (or a source) degenerative inductor. For the common-emitter amplifier with shunt-shunt capacitive feedback, shown in Fig. 2(b), suppose $\left(1+G_{M 1} R_{L}\right) \gg\left|s R_{L} C_{T}\right|$ over the $3.1-10.6-\mathrm{GHz}$ band of interest, which is usually the case. The input impedance $Z_{\mathrm{IN}}$ of it is then given by

$$
Z_{\mathrm{IN}}=s L_{G M}+R_{\mathrm{IN}}+\frac{1}{s C_{\mathrm{IN}}}
$$

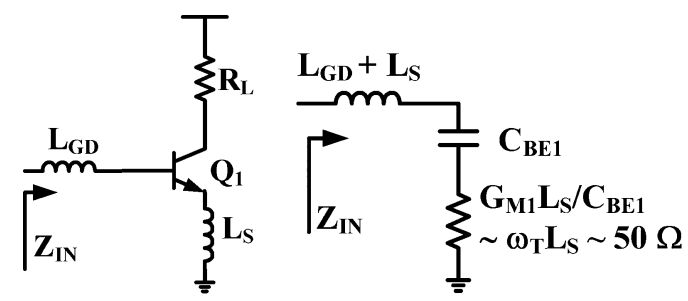

(a)

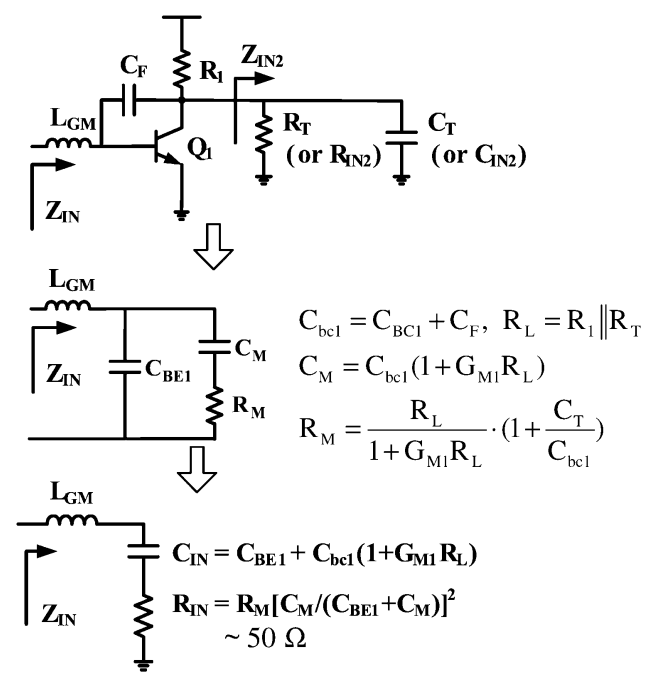

(b)

Fig. 2. Input impedance matching achieved by the: (a) traditional series-series inductive feedback and (b) shunt-shunt capacitive feedback.

in which

$$
\begin{aligned}
C_{\mathrm{IN}} & =C_{\mathrm{BE} 1}+C_{M}=C_{\mathrm{BE} 1}+C_{\mathrm{bc} 1}\left(1+G_{M 1} R_{L}\right) \\
R_{\mathrm{IN}} & =R_{M}\left(\frac{C_{M}}{C_{\mathrm{BE} 1}+C_{M}}\right)^{2} \approx 50 \Omega \\
R_{M} & =\frac{R_{L}}{1+G_{M 1} R_{L}} \cdot\left(1+\frac{C_{T}}{C_{\mathrm{bc} 1}}\right) \\
R_{L} & =R_{1} \| R_{T} .
\end{aligned}
$$

In the above equations, $G_{M 1}$ is the transconductance and $C_{\mathrm{BE} 1}$ is the base-emitter capacitance of transistor $Q_{1} . C_{\mathrm{bc} 1}$ is the equivalent base-collector capacitance, which can include the base-collector capacitance $\left(C_{\mathrm{BC} 1}\right)$ and the intentionally added capacitance $\left(C_{F}\right)$, if necessary, between the base and collector of transistor $Q_{1}$. The parallel $R_{T}-C_{T}$ load represents the input impedance of the following stages of the circuit, which provides an appropriate load for generating the necessary $50 \Omega$. Compared with the traditional series-series inductive input matching technique (two inductors), the shunt-shunt capacitive input matching method (one inductor) is more area efficient because no area consuming inductor $\left(L_{s}\right)$ is needed to generate $50 \Omega$.

\section{B. UWB Gain Flattening Technique}

As shown in Fig. 3, resistive shunt-shunt feedback is traditionally adopted for extending the bandwidth of an amplifier. However, the corresponding gain performance is usually not 


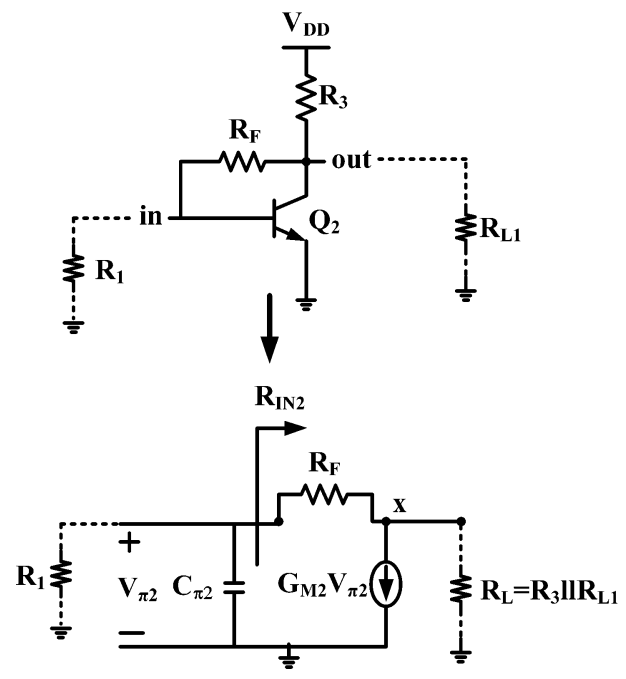

Fig. 3. Traditional resistive shunt-shunt feedback amplifier and its equivalent circuit. The loading effect of the previous stage $\left(R_{1}\right)$ and the output terminal $\left(R_{L 1}\right)$ is also shown.

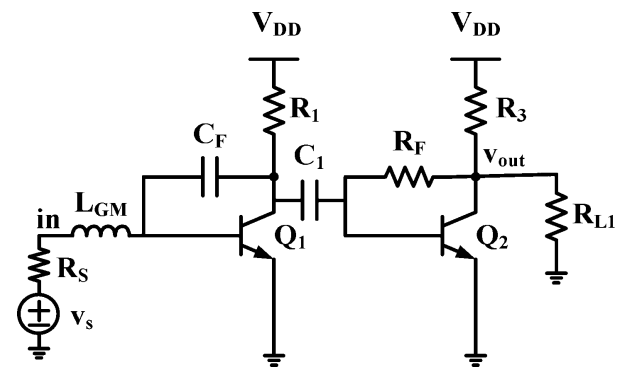

Fig. 4. Multiband LNA consists of a shunt-shunt capacitive feedback stage followed by a gain stage.

high enough for UWB application due to the tradeoff between gain and bandwidth. The transimpedance gain $Z_{\mathrm{RFB}}$ and the pole $P_{1}$ arising from $C_{\pi 2}$ of the resistive shunt-shunt feedback amplifier can be expressed as follows:

$$
\begin{aligned}
Z_{\mathrm{RFB}} & =-\frac{R_{\mathrm{IN} 2} \| R_{1}}{1+\frac{s}{P_{1}}} \times\left(G_{M 2}-\frac{1}{R_{F}}\right)\left(R_{F} \| R_{L}\right) \\
P_{1} & =\frac{1}{C_{\pi 2}\left[R_{1} \| R_{\mathrm{IN} 2}\right]} \\
& =\frac{1}{C_{\pi 2} \times\left[R_{1} \|\left(\frac{R_{F}+R_{L}}{1+G_{M 2} R_{L}}\right)\right]}
\end{aligned}
$$

where $G_{M 2}$ is the transconductance of transistor $Q_{2}, \|$ denotes parallel connection, $R_{L}=R_{3} \| R_{L 1}$, and $R_{\mathrm{IN} 2}=$ $\left(R_{F}+R_{L}\right) /\left(1+G_{M 2} R_{L}\right)$ is the input resistance of the amplifier without considering $C_{\pi 2}$. Note that the loading effect of the previous stage (i.e., $R_{1}$ in Fig. 3 ) and the output terminal (i.e., $R_{L 1}$ in Fig. 3) is included so that the result can be applied directly to the analysis of $S_{21}$ of the amplifier shown in Fig. 4. From (6) and (7), we can see that decreasing $R_{F}$ widens the bandwidth of the amplifier, but at the same time, the gain is degraded.

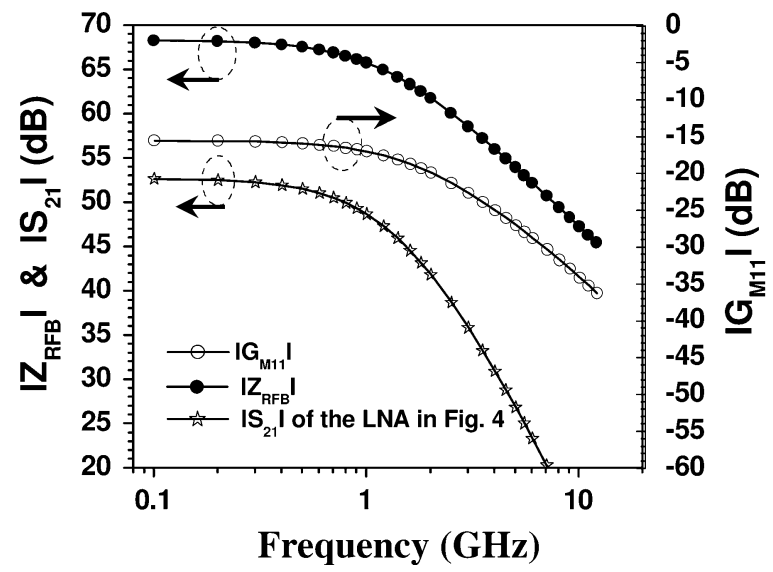

Fig. 5. Calculated characteristics of $\left|G_{M 11}\right|,\left|Z_{\mathrm{RFB}}\right|$, and $\left|S_{21}\right|$ versus frequency of a SiGe multiband LNA.

Although this circuit topology in conjunction with the abovementioned capacitive feedback technique is appropriate as a multiband LNA (see Fig. 4) for a multiband or multistandard application [18], [19], it is difficult to achieve high and flat UWB gain at the same time, which is explained as follows. For the multiband LNA shown in Fig. 4, the transfer function of $S_{21}$, which is equal to two times of the voltage gain $\left(A_{V}=v_{\text {out }} / v_{s}\right)$, can be derived as follows:

$$
\begin{aligned}
S_{21}= & 2 \frac{v_{\mathrm{out}}}{v_{s}} \\
\approx & 2 \frac{\frac{1}{s C_{\mathrm{IN}}}}{R_{S}+s L_{G M}+\frac{1}{s C_{\mathrm{IN}}}+R_{\mathrm{IN}}} \\
& \cdot\left(G_{M 1}-s C_{\mathrm{bc} 1}\right) \cdot\left(R_{1}\left\|R_{\mathrm{IN} 2}\right\| \frac{1}{s\left(C_{\pi 2}+C_{\mathrm{bc} 1}\right)}\right) \\
& \cdot\left(G_{M 2}-\frac{1}{R_{F}}\right) \cdot\left(R_{F} \| R_{L}\right) \\
\equiv & \frac{2 \cdot G_{M 1}}{s^{2} L_{G M} C_{\mathrm{IN}}+1+s C_{\mathrm{IN}}\left(R_{\mathrm{IN}}+R_{s}\right)} \cdot Z_{\mathrm{RFB}} \\
\equiv & G_{M 11} \cdot Z_{\mathrm{RFB}}
\end{aligned}
$$

in which $G_{M 11}$ is proportional to $1 /\left(s^{2} L_{G M} C_{\mathrm{IN}}+1+\right.$ $\left.s C_{\mathrm{IN}}\left(R_{\mathrm{IN}}+R_{s}\right)\right)$, which is a transfer function of a second-order low- $Q$ low-pass $R L C$ circuit. For example, $Q_{\mathrm{IN}}=\sqrt{L_{G M} / C_{\mathrm{IN}}} /\left(R_{\mathrm{IN}}+R_{s}\right) \approx 0.22$, i.e., over-damped response, if $L_{G M}=0.6 \mathrm{nH}$ and the center frequency $f_{o}=5.7 \mathrm{GHz}$ over the band of interest are specified. Besides, the zero $\left(=G_{M 1} /\left(2 \pi C_{\mathrm{bc} 1}\right) \approx 122.5 \mathrm{GHz}\right)$ contributed by the capacitive-feedback first-stage is much higher than the 3.1-10.6-GHz band of interest and, hence, is negligible.

Fig. 5 shows the characteristic of calculated $\left|G_{M 11}\right|,\left|Z_{\mathrm{RFB}}\right|$ and $\left|S_{21}\right|$ versus frequency of a typical SiGe multiband LNA, as published in [19]. As can be seen, over the 3.1-10.6-GHz band of interest, the characteristic of $\left|Z_{\mathrm{RFB}}\right|$ versus frequency exhibits a negative slope close to $20 \mathrm{~dB} /$ decade because its pole is approximately $1.2 \mathrm{GHz}$. In addition, the characteristic of $\left|G_{M 11}\right|$ versus frequency also exhibits a negative slope close to $20 \mathrm{~dB} /$ decade, which can be explained as follows: since 


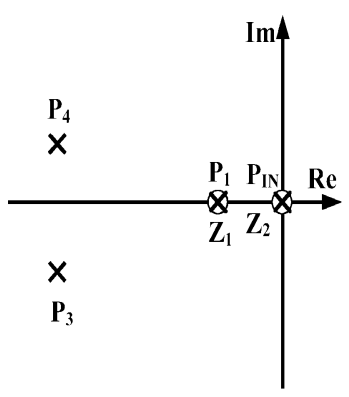

(a)

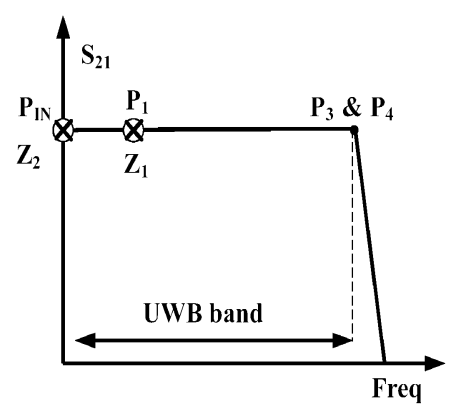

(b)
Fig. 6. (a) Relative position of the zeros $\left(Z_{1}\right.$ and $\left.Z_{2}\right)$ and the poles $\left(P_{1}, P_{3}, P_{4}\right.$, and $\left.P_{\mathrm{IN}}\right)$. (b) Corresponding frequency response of the UWB LNA proposed in this paper.

the input $R L C$ circuit is a low- $Q$ circuit and $f_{o}$ is specified as $5.7 \mathrm{GHz}, s^{2} L_{G M} C_{\mathrm{IN}}+1 \approx 0$ holds over the band of interest, i.e., $s^{2} L_{G M} C_{\mathrm{IN}}+1+s C_{\mathrm{IN}}\left(R_{\mathrm{IN}}+R_{s}\right) \approx s C_{\mathrm{IN}}\left(R_{\mathrm{IN}}+R_{s}\right)$ can be assumed, which, in turn, results in

$$
\begin{aligned}
G_{M 11} & \equiv \frac{2 \cdot G_{M 1}}{s^{2} L_{G M} C_{\mathrm{IN}}+1+s C_{\mathrm{IN}}\left(R_{\mathrm{IN}}+R_{s}\right)} \\
& \approx \frac{2 \cdot G_{M 1}}{s C_{\mathrm{IN}}\left(R_{\mathrm{IN}}+R_{s}\right)} .
\end{aligned}
$$

This explains why the characteristic of $\left|G_{M 11}\right|$ and $\left|S_{21}\right|$ versus frequency in Fig. 5 exhibits a negative slope close to 20 and $40 \mathrm{~dB} / \mathrm{decade}$, respectively, over the $3.1-10.6-\mathrm{GHz}$ band of interest.

To flatten the $S_{21}$ performance of the LNA in Fig. 4 for UWB applications, the poles contributed by $G_{M 11}$ [see $P_{\mathrm{IN}} \sim 0$ in Fig. 6(a) and (b)] and $Z_{\mathrm{RFB}}$ [see $P_{1}$ in Fig. 6(a) and (b)], respectively, should be cancelled by introducing an additional two zeros [see $Z_{2}$ and $Z_{1}$ in Fig. 6(a) and (b)], which are located exactly at the same frequencies of the two poles.

This can be achieved by taking the following two actions. First, adding an inductive shunt-shunt feedback stage (see Fig. 7, in which $L_{1}$ is the feedback inductance and $R_{4}$ is the parasitic resistance of $L_{1}$ or the intentionally added small resistance) after the $Q_{2}$ gain stage of the LNA. A newly generated zero $\left(Z_{1}\right)$ due to the inductive shunt-shunt feedback stage is specified to be equal to the pole $\left(P_{1}\right)$ of $Z_{\mathrm{RFB}}$ to achieve pole-zero cancellation.

Second, adding a stage with an inductive load [see $L_{2}$ in Fig. 8(a)] to the end of the LNA to generate a zero $\left(Z_{2}\right)$ at dc frequency so that the pole contributed by $G_{M 11}\left(P_{\mathrm{IN}} \sim 0\right)$ can

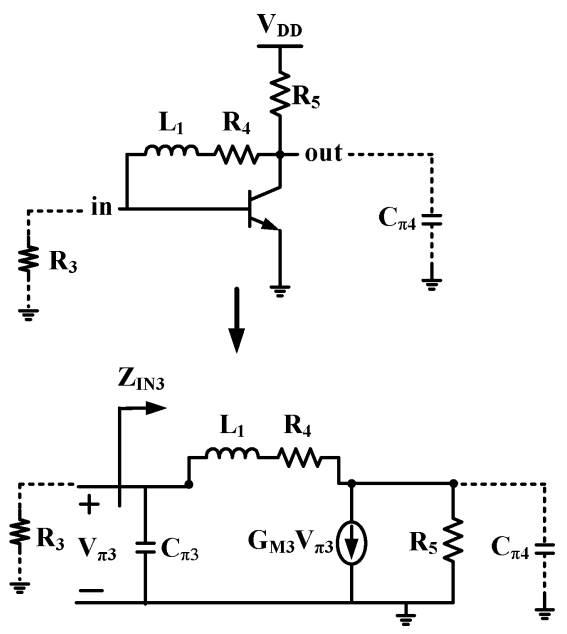

Fig. 7. Proposed inductive shunt-shunt feedback amplifier and its equivalent circuit. The loading effect of the previous stage $\left(R_{3}\right)$ and the next stage $\left(C_{\pi 4}\right)$ is also considered.

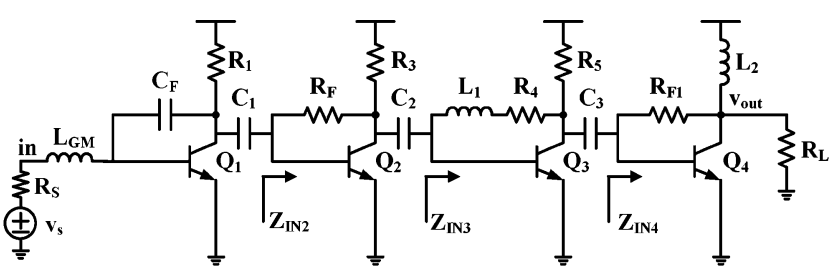

(a)

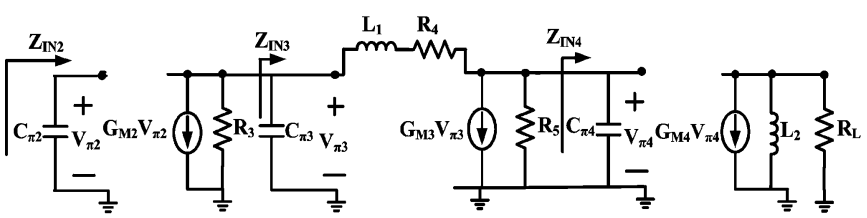

(b)

Fig. 8. (a) Schematic of the proposed UWB LNA. (b) Corresponding equivalent circuit for calculating the input impedances seen at the input of the second, third, and last stages.

be cancelled. Consequently, because frequencies of the complex conjugate poles $\left(P_{3}\right.$ and $P_{4}$, which will be discussed in detail later) arising from the inductive shunt-shunt feedback stage are higher than that of $P_{1}$ originated from the $Q_{2}$ gain stage, the bandwidth of the LNA is extended.

In the following, we analyze the proposed inductive shunt-shunt feedback amplifier shown in Fig. 7. The transimpedance gain $Z_{\mathrm{LFB}}$ and input impedance $Z_{\mathrm{IN} 3}$ can be derived as (10a), shown at the bottom of this page, and (10b),

$$
\begin{aligned}
Z_{\mathrm{LFB}} & =-\left(R_{3} \| Z_{\mathrm{IN} 3}\right)\left(G_{M 3}-\frac{1}{s L_{1}+R_{4}}\right)\left(\left(R_{4}+s L_{1}\right)\left\|R_{5}\right\| \frac{1}{s C_{\pi 4}}\right) \\
& \approx-\frac{R_{3} G_{M 3}\left(R_{4}+s L_{1}\right) R_{5}}{R_{3}\left[\left(1+s C_{\pi 4} R_{5}\right)+G_{M 3} R_{5}\right]+\left[\left(1+s C_{\pi 4} R_{5}\right)\left(R_{4}+s L_{1}\right)+R_{5}\right]+s C_{\pi 3} R_{3}\left[\left(1+s C_{\pi 4} R_{5}\right)\left(R_{4}+s L_{1}\right)+R_{5}\right]}
\end{aligned}
$$


respectively, where $G_{M 3}$ is the transconductance of transistor $Q_{3}$,

$$
Z_{\mathrm{IN} 3}=\frac{\left(s L_{1}+R_{4}\right)+\left(R_{5} \| \frac{1}{s C_{\pi 4}}\right)}{1+G_{M 3} \cdot\left(R_{5} \| \frac{1}{s C_{\pi 4}}\right)} \| \frac{1}{s C_{\pi 3}} .
$$

Note that in (10a), $G_{M 3} R_{4} \gg 1$ is assumed. In addition, the loading effect of the previous stage (i.e., $R_{3}$ in Fig. 7) and the next stage (i.e., $C_{\pi 4}$ in Fig. 7) is included so that the result can be applied directly to the analysis of the $S_{21}$ of the proposed UWB LNA in Section III. Clearly, (10a) has one zero $\left(Z_{1}\right)$ given by

$$
Z_{1}=\frac{R_{4}}{L_{1}}
$$

Note that all the poles and zeros in this paper are located at the left half-plane (LHP). However, we omit the minus sign of them for the purpose of simplification. As mentioned above, $Z_{1}$ is set to be equal to $P_{1}$ in (7) to achieve pole-zero cancellation. Thus, the $\left|S_{21}\right|$ performance of the cascaded amplifier, which consists of a gain stage followed by an inductive shunt-shunt feedback stage, is flat for frequencies up to some pole frequency of the inductive shunt-shunt feedback stage, if a maximally flat response is chosen.

In the following, we discuss the poles of (10a). The characteristic equation of (10a) can be written as follows:

$$
\begin{aligned}
s^{3}+ & s^{2}\left(\omega_{P 2}+\omega_{P 3}+\frac{R_{4}}{L_{1}}\right) \\
& +s\left(\frac{\left(R_{4}+R_{5}\right) \omega_{P 2}}{L_{1}}+\omega_{P 2} \omega_{P 3}+\frac{\left(R_{3}+R_{4}\right) \omega_{P 3}}{L_{1}}\right) \\
& +\left[\frac{\omega_{P 2} \omega_{P 3}}{L_{1}}\left(R_{3}+R_{4}+R_{5}\left(1+G_{M 3} R_{3}\right)\right)\right] \\
\equiv & \left(s+P_{2}\right) \cdot\left(s+P_{3}\right) \cdot\left(s+P_{4}\right) \\
= & 0
\end{aligned}
$$

in which $\omega_{P 2} \equiv 1 /\left(C_{\pi 4} R_{5}\right)$ and $\omega_{P 3} \equiv 1 /\left(C_{\pi 3} R_{3}\right)$ are the higher and lower frequency poles, respectively, of the open-loop amplifier, i.e., $L_{1} \rightarrow \infty$. This is because all the terms on the left side of (12), which include $L_{1}$, are equal to zero if $L_{1} \rightarrow \infty . P_{2}$ and $P_{3}$ are the corresponding poles of $\omega_{P 2}$ and $\omega_{P 3}$, respectively, if the effect of $L_{1}$ is considered. $P_{4}$ is a newly generated pole if the effect of $L_{1}$ is considered. Note that $P_{4}$ will be very close to the origin if $L_{1}$ is very large. From the coefficients of the second-order and constant terms of (12), we can obtain the following equations:

$$
\begin{aligned}
\omega_{P 2}+\omega_{P 3}+Z_{1} & =P_{2}+P_{3}+P_{4} \\
\frac{\omega_{P 2} \omega_{P 3}}{L_{1}}\left(R_{3}+R_{4}+R_{5}\left(1+G_{M 3} R_{3}\right)\right) & =P_{2} \cdot P_{3} \cdot P_{4} .
\end{aligned}
$$

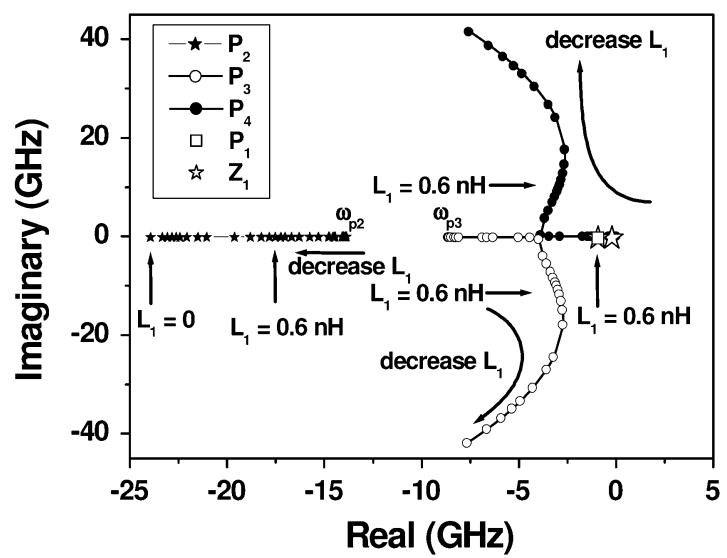

Fig. 9. Calculated root-locus diagram of the inductive shunt-shunt feedback amplifier in Fig. 8 by (10a) using the circuit parameters from the SiGe HBT UWB LNA.

Equation (13) implies that if a very large $L_{1}$ is added to the open loop amplifier, both $Z_{1}$ and $P_{4}$ will be created simultaneously around the origin since $P_{2} \approx \omega_{P 2}, P_{3} \approx \omega_{P 3}$, and $P_{4} \approx 0$. This is consistent with the calculated root-locus diagram shown in Fig. 9. Furthermore, since $P_{2}$ and $P_{3}$ are very close to $\omega_{P 2}$ and $\omega_{P 3}$, respectively, for a very large $L_{1}, P_{4}$ can be approximated by the following equation based on (14):

$$
P_{4}=\frac{R_{3}+R_{4}+R_{5}\left(1+G_{M 3} R_{3}\right)}{L_{1}} .
$$

Equation (15) predicts that the movement of $P_{4}$ to the left will be quicker than that of $Z_{1}$ to the left as the value of $L_{1}$ is reduced, which is indeed the case, as shown in Fig. 9. As indicated, decreasing $L_{1}$ from infinity moves the highest frequency pole $\left(P_{2}\right)$ outward, while the other poles $\left(P_{3}\right.$ and $\left.P_{4}\right)$ become coincident and then become complex and conjugate. The inductive shunt-shunt feedback amplifier used in this paper belongs to the latter situation (i.e., $P_{3}$ and $P_{4}$ are complex and conjugate) because a small value of $L_{1}(0.6 \mathrm{nH})$ is adopted.

It is interesting to note that if the value of $R_{4}$ is negligible and the value of $L_{1}$ is 0 , corresponding to the case of shortening transistor base and collector terminals, then (12) can be simplified as follows:

$$
s=-\frac{1}{\left(C_{\pi 3}+C_{\pi 4}\right)\left(R_{3}\left\|R_{5}\right\| \frac{1}{G_{M 3}}\right)}
$$

which is consistent with the result obtained by direct circuit analysis and also explains why $P_{2}$ approaches to a limiting frequency, as is evident in Fig. 9.

\section{PRoposed UWB LNA}

\section{A. Circuit Topology}

Fig. 8(a) shows the proposed UWB LNA with four cascaded stages, including a common-emitter amplifier with Miller capacitance feedback for wideband input impedance matching, 
a common-emitter gain stage, an inductive shunt-shunt feedback amplifier for bandwidth extension by pole-zero cancellation, and a buffer amplifier with an inductive load $L_{2}$. Large resistances $R_{F}$ and $R_{F 1}$ were used for self-biasing and $C_{1}, C_{2}$, and $C_{3}$ were used as dc blocking capacitors.

The equivalent loading impedance $\left(Z_{\mathrm{IN} 2} \| R_{1}\right)$ of the first stage provides an appropriate load for generating the necessary $50 \Omega$ by the beneficial Miller capacitance $C_{\mathrm{bc} 1} \cdot\left(1+G_{M 1} R_{L}\right)$. Apart from the purpose of generating $50 \Omega$, the Miller capacitance is also used to enlarge the input capacitance $C_{\mathrm{IN}}$ of the input low- $Q R L C$ circuit [see (5b)], which, in turn, results in a larger input matching bandwidth. Fig. 8(b) shows the equivalent circuit of the second to the fourth stage of the proposed UWB LNA for calculating the $Z_{\mathrm{IN} 4}, Z_{\mathrm{IN} 3}$, and $Z_{\mathrm{IN} 2}$, which can be expressed, respectively, as follows:

$$
\begin{aligned}
Z_{\mathrm{IN} 4} & =\frac{1}{s C_{\pi 4}} \\
Z_{\mathrm{IN} 3}= & \frac{s L_{1}+R_{4}+\left(R_{5} \| Z_{\mathrm{IN} 4}\right)}{1+G_{M 3} \cdot\left(R_{5} \| Z_{\mathrm{IN} 4}\right)} \| \frac{1}{s C_{\pi 3}} \\
= & \frac{s L_{1}+R_{4}+\left(R_{5} \| \frac{1}{s C_{\pi 4}}\right)}{1+G_{M 3} \cdot\left(R_{5} \| \frac{1}{s C_{\pi 4}}\right)} \| \frac{1}{s C_{\pi 3}} \\
Z_{\mathrm{IN} 2} & =\frac{1}{s C_{\pi 2}} .
\end{aligned}
$$

In deriving the above equations, the two large self-biased resistors $R_{F}$ and $R_{F 1}$ have been neglected. $S_{21}$ of the four-stage cascaded UWB LNA, which is equal to two times of the voltage gain $\left(A_{V}=v_{\text {out }} / v_{s}\right)$, can be expressed as (18a) and (18b), shown at the bottom of this page, where $R_{\mathrm{IN}}\left(=R_{S}=R_{L}=\right.$ $50 \Omega)$ and $C_{\mathrm{IN}}$ are the equivalent input circuit elements seen at the base of $Q_{1}$ with the capacitive feedback input matching method and

$$
\begin{aligned}
& \left.Z_{L 1}=Z_{\mathrm{IN} 2}\left\|R_{1}\right\|\left(1 /\left(s C_{\mathrm{bc} 1}\right)\right) \approx\left(1 / s C_{\pi 2}\right) \| R_{1}\right) \\
& Z_{L 2}=Z_{\mathrm{IN} 3} \| R_{3} \\
& Z_{L 3}=Z_{\mathrm{IN} 4}\left\|R_{5}\right\|\left(s L_{1}+R_{4}\right)
\end{aligned}
$$

are the loading impedances, with the input impedance of the next stage being considered, of the first, second, and third stages, respectively.

Equation (18b) can be further discussed as follows. First, in order to achieve UWB input impedance matching, the series $L_{G M}-C_{\mathrm{IN}}-R_{\mathrm{IN}}$ input equivalent circuit of the UWB LNA is designed as a low- $Q$ circuit, which is different from that (high- $Q L-C-R$ input equivalent circuit) used in the narrowband LNAs [17]. Since the input $R L C$ circuit is a low- $Q$ circuit and its center frequency $f_{o}$ can be specified as the geometrical mean $(5.7 \mathrm{GHz})$ of the upper $(10.6 \mathrm{GHz})$ and lower (3.1 GHz) corner frequency, $s^{2} L_{G M} C_{\mathrm{IN}}+1+s C_{\mathrm{IN}}\left(R_{\mathrm{IN}}+\right.$ $\left.R_{s}\right) \approx s C_{\mathrm{IN}}\left(R_{\mathrm{IN}}+R_{S}\right)$ holds due to $s^{2} L_{G M} C_{\mathrm{IN}}+1 \approx 0$. This means that the pole $\left(P_{\mathrm{IN}} \sim 0\right)$ of $s^{2} L_{G M} C_{\mathrm{IN}}+1+s C_{\mathrm{IN}}\left(R_{\mathrm{IN}}+\right.$ $\left.R_{s}\right) \approx s C_{\mathrm{IN}}\left(R_{\mathrm{IN}}+R_{s}\right)$ can be cancelled by the zero $\left(Z_{2}\right)$ contributed by the impedance $\left(s L_{2}\right)$ of $L_{2}$. Moreover, the zero $\left(Z_{1}\right)$ of $Z_{\mathrm{LFB}}$ is set to be equal to the pole $\left(P_{1}\right)$ of $Z_{\mathrm{RFB}}$, i.e., $L_{1} \approx R_{4} R_{1} C_{\pi 2}$, to achieve pole-zero cancellation. If the pole originates from the addition of $L_{2}$ [i.e., $1 /\left(R_{L}+s L_{2}\right)$ in (18b)] is negligible (i.e., larger than $10.6 \mathrm{GHz}$ ), then the maximally flat response condition for $P_{3}$ and $P_{4}$ should be chosen. If the pole originating from the addition of $L_{2}$ cannot be neglected (i.e., smaller than $10.6 \mathrm{GHz}$ ), then a small peaking can be chosen to compensate the frequency dependency so that a flat response of $S_{21}$ can still be achieved. It is interesting to note that the circuit architecture of the third stage of the UWB LNA, which is inductive feedback, is similar to that of a Colpitts oscillator [20] if the small feedback resistance $R_{4}$ is neglected. That means, in general, an inductive-feedback peaking extends the bandwidth of an amplifier at the expense of stability. Fig. 6(a) shows the relative position of the zeros $\left(Z_{1}\right.$ and $\left.Z_{2}\right)$ and poles $\left(P_{1}, P_{3}, P_{4}\right.$, and $\left.P_{\mathrm{IN}}\right)$ according to the proposed methodology. The corresponding frequency response of the UWB LNA is illustrated in Fig. 6(b).

An SiGe HBT UWB LNA was designed with a standard $0.35-\mu \mathrm{m}$ SiGe HBT process based on the above-mentioned circuit design principle. The component parameters are as follows: $L_{G M}=0.6 \mathrm{nH}, L_{1}=0.6 \mathrm{nH}, L_{2}=2.46 \mathrm{nH}$, $R_{1}=300 \Omega, R_{F}=2010 \Omega, R_{3}=125 \Omega, R_{4}=3 \Omega, R_{5}=$ $101 \Omega, R_{F 1}=1000 \Omega, C_{1}=3 \mathrm{pF}, C_{2}=3 \mathrm{pF}, C_{3}=3 \mathrm{pF}$, and $C_{F}=18 \mathrm{fF}$. All transistors $\left(Q_{1}, Q_{2}, Q_{3}\right.$, and $\left.Q_{4}\right)$ have the

$$
\begin{aligned}
S_{21}= & 2 A_{V} \\
= & 2 \frac{v_{\mathrm{out}}}{v_{s}} \\
\approx & 2 \frac{\frac{1}{s C_{\mathrm{IN}}}}{R_{S}+s L_{G M}+\frac{1}{s C_{\mathrm{IN}}}+R_{\mathrm{IN}}} \cdot G_{M 1} \cdot Z_{L 1} \cdot G_{M 2} \cdot Z_{L 2} \cdot\left(G_{M 3}-\frac{1}{s L_{1}+R_{4}}\right) \cdot Z_{L 3} \cdot G_{M 4} \cdot \frac{R_{L} s L_{2}}{R_{L}+s L_{2}} \\
\approx & 2 \frac{1}{s^{2} L_{G M} C_{\mathrm{IN}}+1+s\left(R_{S}+R_{\mathrm{IN}}\right) C_{\mathrm{IN}}} \cdot G_{M 1} \cdot \frac{R_{1}}{1+s R_{1} C_{\pi 2}} \cdot G_{M 2} \cdot G_{M 4} \cdot \frac{R_{L} \cdot s L_{2}}{R_{L}+s L_{2}} \\
& \cdot \frac{R_{3} G_{M 3}\left(R_{4}+s L_{1}\right) R_{5}}{R_{3}\left[\left(1+s C_{\pi 4} R_{5}\right)+G_{M 3} R_{5}\right]+\left[\left(1+s C_{\pi 4} R_{5}\right)\left(R_{4}+s L_{1}\right)+R_{5}\right]+s C_{\pi 3} R_{3}\left[\left(1+s C_{\pi 4} R_{5}\right)\left(R_{4}+s L_{1}\right)+R_{5}\right]}
\end{aligned}
$$




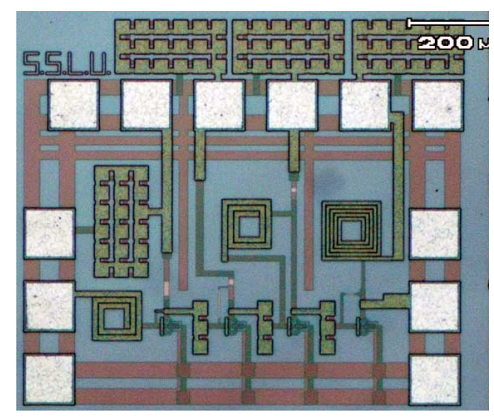

Fig. 10. Die photograph of the SiGe HBT UWB LNA.

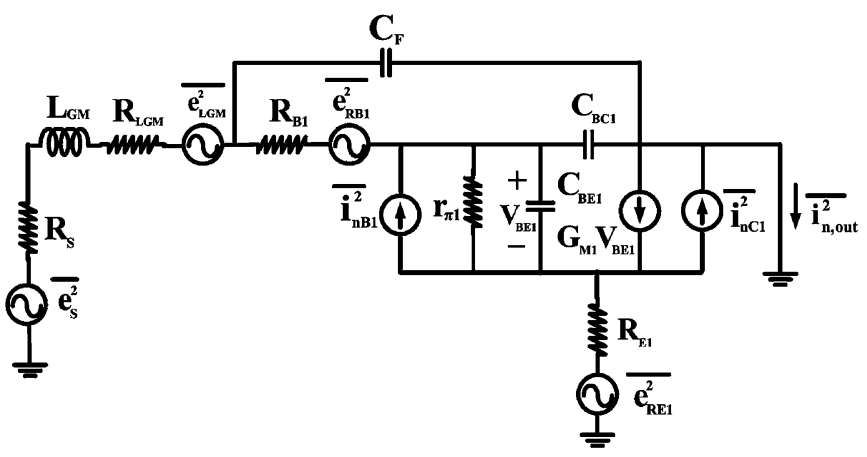

Fig. 11. Complete equivalent circuit of Fig. 2(b) for noise calculation.

same emitter size of $0.3 \mu \mathrm{m} \times 20.3 \mu \mathrm{m} \times 1$ emitter finger. The die photograph of the finished circuit is shown in Fig. 10. The chip area is only $570 \mu \mathrm{m} \times 390 \mu \mathrm{m}$ excluding the test pads.

\section{B. Noise Analysis}

According to the Friis equation [21], the total NF is dominated by the NF of the first stage if the gain of the first stage is high enough. Fig. 11 shows the equivalent circuit of the first stage of the proposed UWB LNA for first-order approximation of noise calculation. Based on our previous study [17], the NF of the first stage can be given by (20), shown at the bottom of this page, where $R_{S}$ is the signal source resistance (usually $50 \Omega), R_{E 1} / R_{B 1}$ is the parasitic emitter/base resistance of transistor $Q_{1}, R_{L G M}$ is the series resistance of the inductor $L_{G M}$, $k$ is the Boltzmann constant, $T$ is absolute temperature, $f$ is frequency, $i_{n B 1}$ is the base noise current, and $i_{n C 1}$ is the collector noise current.

Note that the capacitance term $\left(C_{\mathrm{BE} 1}+C_{\mathrm{bc} 1}\right)$ of (20) is different from that $\left(C_{\mathrm{BE} 1}+C_{\mathrm{bc} 1} \cdot\left(1+G_{M 1} R_{L}\right)\right)$ of $S_{11}$ [see (2) and (5b)] unless $C_{\mathrm{bc} 1}$ is small, assuming $C_{\mathrm{bc} 1}=0$ and $R_{S}+R_{L G M}+R_{B 1}+R_{E 1} \approx R_{S}$. To gain more insight into
(20), let us temporarily neglect the noise contribution from $i_{n B 1}$ and, hence, (20) can be rewritten as

$$
\begin{aligned}
\mathrm{NF} \approx & 1+\frac{R_{L_{G M}}+R_{B 1}+R_{E 1}}{R_{S}}+\frac{1}{2 G_{M 1} R_{S}} \\
& \quad\left|\left(\frac{s}{\omega_{\text {Onf }}}\right)^{2}+s \frac{\left(R_{S}+R_{\mathrm{PAR}}\right)}{Q_{\mathrm{IN}} \cdot \omega_{\text {Onf }} \cdot\left(R_{S}+R_{\mathrm{IN}}+R_{\mathrm{PAR}}\right)}+1\right|^{2}
\end{aligned}
$$

where $\omega_{\text {onf }}=1 / \sqrt{L_{G M} C_{\mathrm{BE} 1}}$. We can see that the numerator of the third term of (21) is similar to the square of the first denominator of the $S_{21}$ expression in (18b) [or $S_{11}$ expression in (2)]. From this, we conclude that the frequency response of the $\mathrm{NF}$, in general, follows the inverse of the gain response of the input $R L C$ circuit. That is, a low- $Q$ circuit (over-damped) wideband input $R L C$ circuit will yield a wideband NF response such as those in [1] and [18], while a high- $Q$ (under-damped) narrowband input $R L C$ circuit will yield a narrowband NF response such as the LNA in [22]. This general trend is true even if $C_{\mathrm{bc} 1}$ is taken into account.

For a low- $Q$ input $R L C$ circuit, $s^{2} L_{G M} C_{\mathrm{IN}} \approx 0$ holds over the band of interest and, hence, (20) can be approximated without considering $i_{n B 1}$ by

$$
\begin{aligned}
\mathrm{NF} \approx & 1+\frac{R_{L G M}+R_{B 1}+R_{E 1}}{R_{S}} \\
& +\frac{1}{2 G_{M 1} R_{S}} \cdot \mid 1+j \omega\left(C_{\mathrm{BE} 1}+C_{\mathrm{bc} 1}\right) \cdot R_{S} \\
& -\left.\omega^{2}\left(C_{\mathrm{BE} 1}+C_{\mathrm{bc} 1}\right) \cdot L_{G M}\right|^{2} \\
\approx & 1+\frac{R_{L G M}+R_{B 1}+R_{E 1}}{R_{S}} \\
& +\frac{R_{S}}{2 G_{M 1}} \cdot\left(C_{\mathrm{BE} 1}+C_{\mathrm{bc} 1}\right)^{2} \cdot \omega^{2} .
\end{aligned}
$$

From (22), we can see that the NF increases with increasing $C_{\mathrm{BE} 1}+C_{\mathrm{bc} 1}$; i.e., in order to reduce the NF, it is better to reduce $C_{\mathrm{BE} 1}+C_{\mathrm{bc} 1}$. However, this is in conflict with the purpose of widening the input matching band, which increases with increasing $C_{\mathrm{BE} 1}$ and/or $C_{\mathrm{bc} 1}$. Hence, there is a tradeoff between the input impedance-matching bandwidth and the NF. We can call this phenomenon matching-bandwidth-NF limitation. Fortunately, because of the Miller effect, the needed $C_{F}$ is normally much smaller than $C_{\mathrm{BE} 1}+C_{\mathrm{BC} 1}$. This means the degradation in the NF due to the intentionally added $C_{F}$ is normally not significant. Since the NF in (22) is inversely proportional to $G_{M 1}$ (or $I_{C 1}$ ), power consumption can be increased, if necessary, to remedy the degradation of the NF. Note that, in fact, the total

$$
\begin{aligned}
\mathrm{NF} \approx 1+\frac{R_{L G M}+R_{B 1}+R_{E 1}}{R_{S}} & +\left|R_{S}+R_{L G M}+R_{B 1}+R_{E 1}+j \omega \cdot L_{G M}\right|^{2} \cdot \frac{\overline{\left|i_{n B 1}^{2}\right|}}{4 k T R_{S} \Delta f} \\
& +\left|\frac{1+j \omega\left(C_{\mathrm{BE} 1}+C_{\mathrm{bc} 1}\right) \cdot\left(R_{S}+R_{L G M}+R_{B 1}+R_{E 1}+j \omega \cdot L_{G M}\right)}{G_{M 1}}\right|^{2} \cdot \frac{\overline{\left|i_{n C 1}^{2}\right|}}{4 k T R_{S} \Delta f}
\end{aligned}
$$


$\mathrm{NF}$ also includes the noise contribution from $i_{n B 1}$, which decreases with increasing $C_{\mathrm{BE} 1}+C_{\mathrm{bc} 1}$. Therefore, in real cases, the degradation of the NF due to the increase of $C_{\mathrm{BE} 1}+C_{\mathrm{bc} 1}$ is offset by the decrease of $i_{n B 1}$.

The noise contributions from both $i_{n B 1}$ and $i_{n C 1}$ are now taken into account. Following the power-constrained noise-optimization method proposed by Shaeffer and Lee [23] and assuming $R_{S}+R_{L G M}+R_{B 1}+R_{E 1} \approx R_{S}, \mathrm{NF}$ (20) near resonance (i.e., $\omega_{o}^{2} L_{G M} C_{\mathrm{IN}} \approx 1$ ) can be rewritten as

$$
\begin{aligned}
\mathrm{NF} \approx & 1+\frac{R_{L G M}+R_{B 1}+R_{E 1}}{R_{S}} \\
& +\left(1+\frac{Q_{S}^{2}}{\left(1+\chi\left(1+\left|A_{V 1}\right|\right)\right)^{2}}\right) \frac{G_{M 1} R_{S}}{2 \beta_{\mathrm{dc}}} \\
& +\left\{\left(\frac{\chi\left|A_{V 1}\right|}{1+\chi\left(1+\left|A_{V 1}\right|\right)}\right)^{2}+\frac{(1+\chi)^{2}}{Q_{S}^{2}}\right\} \frac{1}{2 G_{M 1} R_{S}}
\end{aligned}
$$

where $A_{v 1}$ is the voltage gain of the first stage, and $Q_{S}$ and $\chi$ are defined as follows, respectively:

$$
\begin{aligned}
Q_{S} & =\frac{1}{\omega_{o} C_{\mathrm{BE} 1}\left(R_{S}+R_{\mathrm{PAR}}\right)}=\frac{C_{\mathrm{IN}}\left(R_{s}+R_{\mathrm{IN}}+R_{\mathrm{PAR}}\right)}{C_{\mathrm{BE} 1}\left(R_{S}+R_{\mathrm{PAR}}\right)} Q_{\mathrm{IN}} \\
\chi & =\frac{C_{\mathrm{bc} 1}}{C_{\mathrm{BE} 1}} .
\end{aligned}
$$

The typical value of $\chi$ is around $0.1-0.2$. It follows that (23) includes terms that are proportional to $Q_{S}^{2}$, as well as to $1 / Q_{S}^{2}$. Therefore, a minimum NF exists for a particular $Q_{S}$ (or device size) given by

$$
Q_{S, \mathrm{opt}, I_{C 1}}=\sqrt[4]{\beta_{\mathrm{dc}}} \frac{\sqrt{(1+\chi)\left(1+\chi\left(1+A_{V 1}\right)\right)}}{\sqrt{G_{M 1} R_{S}}} .
$$

Similarly, a minimum NF also exists for a particular $G_{M 1}$ (or $\left.I_{C 1}\right)$ representing power consumption because the NF includes terms that are proportional to $G_{M 1}$ (or $I_{C 1}$ ), as well as to $1 / G_{M 1}$ (or $1 / I_{C 1}$ ). The optimum $G_{M 1}$ can be derived as

$$
G_{M 1, \mathrm{opt}}=\frac{\sqrt{\beta_{\mathrm{dc}}}}{R_{S}} \frac{\sqrt{\left(\frac{\chi A_{V 1}}{1+\chi\left(1+A_{V 1}\right)}\right)^{2}+\frac{(1+\chi)^{2}}{Q_{S}^{2}}}}{\sqrt{1+\frac{Q_{S}^{2}}{\left(1+\chi\left(1+A_{V 1}\right)\right)^{2}}}} .
$$

The characteristics of the NF versus $Q_{\mathrm{IN}}$ using the power consumption $\left(I_{C 1}\right.$ or $\left.G_{M 1}\right)$ as a parameter with a typical de gain of 20 are plotted in Fig. 12. Also remember that the requirement of wideband impedance matching sets an upper bound of $Q_{\mathrm{IN}}=0.25$ (see (4), $L_{G M}<0.707 \mathrm{nH}$ for 3.1-10.6- $\mathrm{GHz}$ input matching). Note that a $G_{M 1}$ greater than $G_{M 1 \text {,opt }} \approx 0.22$ degrades the NF at $Q_{\mathrm{IN}}=0.25$ because the noise contribution from $I_{n B 1}$ becomes dominant beyond $G_{M 1, \mathrm{opt}}$.

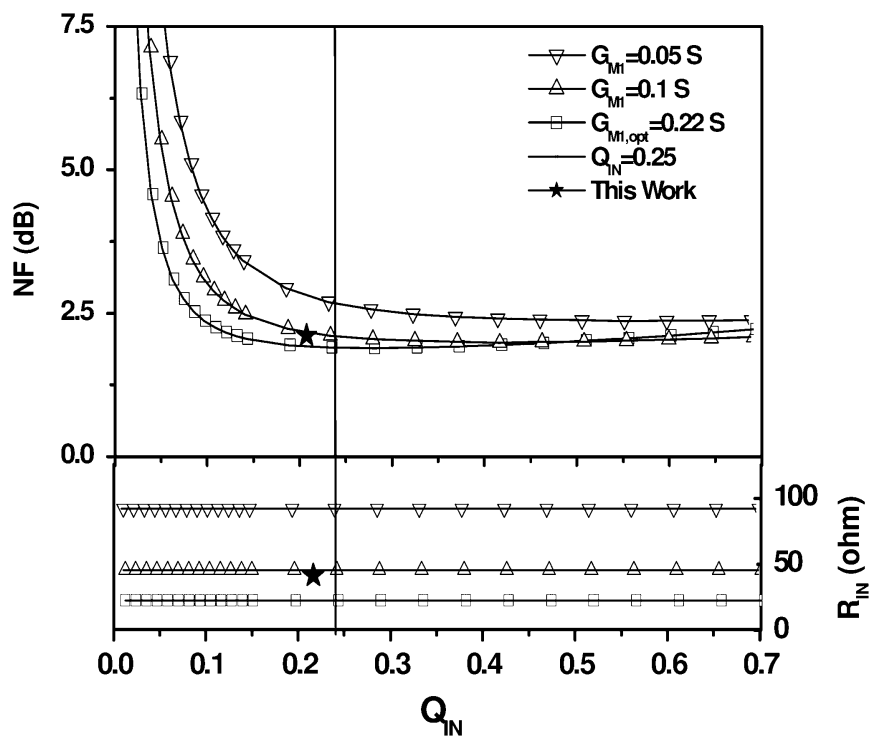

Fig. 12. Characteristics of $\mathrm{NF}$ versus $Q_{\mathrm{IN}}$ using the $G_{M 1}$ as a parameter.

In order to obtain the minimum NF, one should choose $G_{M 1, \text { opt }} \approx 0.22$. However, as is evident in Fig. 12 , the $R_{\mathrm{IN}}$ achieved by setting bias at $G_{M 1 \text {,opt }}$ is usually too small. $R_{\mathrm{IN}}$ generated by feedback capacitance [see (5c)] must be close to $50 \Omega$ and can be approximated by

$$
G_{M 1,50} \approx \frac{1}{50}\left(1+\frac{1}{\chi}\right)
$$

Since $\chi$ is around $0.1-0.2, G_{M 1}$ is near $0.12-0.22$. In this design, $G_{M 1} \approx 0.1$ was chosen for low power consumption. Once $G_{M 1}$ is given, the optimum $Q_{\mathrm{IN}}$ can be determined. Nevertheless, as mentioned previously, $Q_{\mathrm{IN}}$ cannot be greater than 0.25 for wideband input matching.

From Fig. 12, it is clear that the NF is insensitive to $Q_{\mathrm{IN}}$ from 0.15 to 0.7 and, hence, $Q_{\mathrm{IN}}$ of 0.22 was selected because of the available standard inductors provided by the foundry. We thus conclude that the optimum NF, $Q_{\mathrm{IN}}$ (or device size), gain, and power consumption can be determined from Fig. 12. Also note that since (20) was derived from the NF with inductive degeneration, good noise match is also possible with the proposed technique.

\section{Linearity}

In the following, the linearity characteristic and the gain partitioning in the cascaded stages of the LNA are explained. Fig. 13 shows the simulated gain $\left(S_{21}\right)$ of each stage of the amplifier. As can be seen, in the 3-10-GHz band of interest, total gain larger than $25 \mathrm{~dB}$ is obtained. In addition, an average gain of 9.7, 8.8, -4.8 , and $12 \mathrm{~dB}$ is achieved for the first, second, third, and fourth stages, respectively. The high gain of $9.7 \mathrm{~dB}$ in the first stage and $8.8 \mathrm{~dB}$ in the second stage is for achieving reasonable low NF. The low gain of the third stage is for better input third-order intermodulation point (IIP3). Since the bias resistance $R_{F 1}(=1000 \Omega)$ is large enough, the fourth stage of the LNA can be regarded as a typical common-emitter stage without 


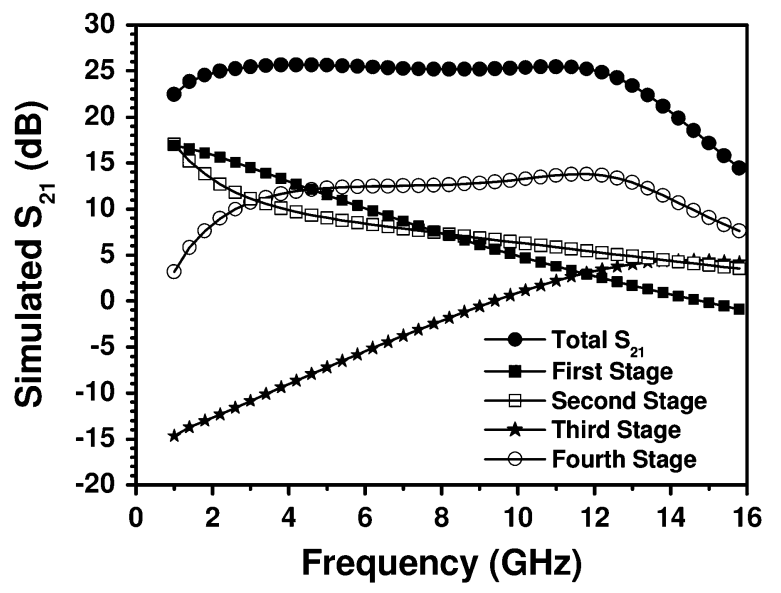

Fig. 13. Simulated $S_{21}$ of the first, second, third, and fourth stages of the SiGe HBT UWB LNA. The overall $S_{21}$ of the LNA is also shown for comparison.

a feedback resistance $R_{F 1}$ between its base and collector terminals. This means IIP3 of the proposed UWB LNA can be approximated as follows [20]:

or

$$
\mathrm{IIP} 3 \approx \frac{\mathrm{IIP} 3,4}{G_{1} \cdot G_{2} \cdot G_{3}} \approx \frac{4 V_{T}^{2} / R_{S 3}}{G_{1} \cdot G_{2} \cdot G_{3}},
$$

$$
\begin{aligned}
\operatorname{IIP} 3(\text { in } \mathrm{dBm}) & \approx \frac{4 V_{T}^{2}}{R_{S 3}}(\text { in } \mathrm{dBm})-G_{1} \cdot G_{2} \cdot G_{3}(\text { in } \mathrm{dB}) \\
& =\frac{4 V_{T}^{2}}{R_{S 3}}(\text { in } \mathrm{dBm})-G(\text { in } \mathrm{dB})+G_{4}(\text { in } \mathrm{dB})
\end{aligned}
$$

in which $G$ is the overall gain of the LNA. $G_{1}, G_{2}, G_{3}$, and $G_{4}$ are the gain of the first, second, third, and fourth stages, respectively, of the LNA. IIP3, 4 is the IIP3 of the fourth stage of the LNA. $R_{S 3}$ is the real part of the input impedance if we look into the output terminal of the third stage, which is equal to $6.28,4.88$, and $1.06 \Omega$, respectively, at $3,5.4$, and $10 \mathrm{GHz}$. Since the LNA exhibits UWB characteristics and the frequency dependence of $R_{S 3}$ is not strong, IIP3 and 1-dB compression point $\left(P_{1 \mathrm{~dB}}\right)$ of the LNA will also exhibit UWB characteristics if $G_{4}$ exhibits UWB characteristics.

\section{MeAsurement Results And Discussions}

The noise and scattering parameters were measured on-wafer using an automated NP5 measurement system from ATN Microwave Inc., North Billerica, MA. The SiGe UWB LNA was biased at $2.74 \mathrm{~mA}$ and $1.5 \mathrm{~V}, 4.88 \mathrm{~mA}$ and $1.7 \mathrm{~V}, 4.3 \mathrm{~mA}$ and $1.7 \mathrm{~V}$, and $6.1 \mathrm{~mA}$ and $1.0 \mathrm{~V}$ for the first, second, third and fourth stages, respectively; i.e., the power consumption is $25.8 \mathrm{~mW}$. As shown in Fig. 14(a), the measured $S_{11}$ is below $-10 \mathrm{~dB}$ for frequency from 3 to $14 \mathrm{GHz}$ (except for a small range from 10 to $11 \mathrm{GHz}$, which is below $-9 \mathrm{~dB}$ ), indicating a very broadband input matching characteristic. This is mainly attributed to the adoption of the capacitive feedback matching technique, which uses the Miller effect to enlarge the input capacitance $C_{\mathrm{IN}}$. Simulation results show that the ripple around $10 \mathrm{GHz}$ originates from the series resonance of inductance
$L_{G M}+L_{1}$ and the equivalent capacitance in the series path [see Fig. 14(a)]. Besides, the simulated $S_{11}$ under both the smalland the large-signal condition (in the presence of $-10-\mathrm{dBm}$ interferer) is also shown in Fig. 14(a). As can be seen, $S_{11}$ is below $-10 \mathrm{~dB}$ from 3 to $14 \mathrm{GHz}$ for both the small- and the large-signal condition. This means the impedance matching still holds even under the large-signal condition. Besides, from the fast-fast (FF) and slow-slow (SS) curves in Fig. 14(a), we can see that the impedance matching in the 3-14-GHz band still holds across standard 3- $\sigma$ process variation.

The measured and calculated $S_{21}$ based on (18b) are shown in Fig. 14(b). As can be seen, the calculated results agree well to the measured data. Peak $S_{21}$ of $26.1 \mathrm{~dB}$ (at $9.6 \mathrm{GHz}$ ) and 3-dB bandwidth of $8.6 \mathrm{GHz}$ (from 3 to $11.6 \mathrm{GHz}$ ) were achieved. In addition, from the FF and SS curves in Fig. 14(b), we can see that the proposed pole-zero cancellation approach is robust enough so under nominal 3- $\sigma$ conditions, the bandwidth is still extended. These results demonstrate that the inductive shunt-shunt feedback technique can indeed achieve high and flat UWB $S_{21}$ characteristics. The four-stage circuit configuration also guarantees a high reverse isolation. The measured $S_{12}$ is below $-42 \mathrm{~dB}$ from 2 to $15 \mathrm{GHz}$ (not shown here), preventing LO leakage signals from transmitting to the antenna.

Fig. 14(c) shows the measured and calculated [by (20)] NF of the SiGe UWB LNA. This LNA achieves an NF of 2.5, 5.8, and $7.1 \mathrm{~dB}$ at 3,10 , and $12 \mathrm{GHz}$, respectively. These values are very close to the calculated results of $2.1,5.5$, and $6.8 \mathrm{~dB}$, respectively, at 3,10 , and $12 \mathrm{GHz}$. The consistency between the measured and calculated ones verifies our noise analysis in Section III. Since the input matching bandwidth in this study (3-14 GHz) is wider than the specification $(3.1-10.6 \mathrm{GHz})$, the $\mathrm{NF}$ frequency response can be flattened by reducing the capacitance term $\left(C_{\mathrm{BE} 1}+C_{\mathrm{bc} 1}\right)$ [see (22)] at the expense of the input matching bandwidth.

A good phase linearity property is achieved, i.e., group-delay variation is only \pm 28 ps across the entire band [see Fig. 14(d)]. By definition, group delay is the derivation of the phase of transfer function $S_{21}$ and, hence, any resonance in the signal path (or pole in $S_{21}$ ) will contribute distortion in the group delay [24]. This means the good group-delay variation characteristic of the proposed UWB LNA architecture is attributed to the pushing of poles $P_{3}$ and $P_{4}$ [see Fig. 6(b)] outside of the $3-10-\mathrm{GHz}$-band of interest by the shunt-shunt feedback inductor. These results again demonstrate that this UWB LNA is very suitable for UWB pulse radio system applications. An expression often used to characterize the stability of an LNA is the Stern stability factor ( $K$-factor), defined in [20]. In addition, it has been shown that $\mu>1$ (or $\mu^{\prime}>1$ alone is necessary and sufficient for a circuit to be unconditionally stable [25]. Fig. 15 shows the measured $K$-factor, $\mu$, and $\mu^{\prime}$ of the SiGe HBT UWB LNA. Clearly, the LNA is unconditionally stable from 2 to $14 \mathrm{GHz}$.

Microwave power performances were measured by a load-pull ATN system with automatic tuners. The LNA achieves IIP3 of $-18,-17$, and $-18 \mathrm{dBm}$ at $3,5.4$, and $10 \mathrm{GHz}$, respectively, which conform well with those $(-18.3,-16.1$, and $-15.7 \mathrm{dBm}$ at $3,5.4$, and $10 \mathrm{GHz}$, respectively) calculated by (29), and close to those $(-23.3,-21.6$, and $-17.8 \mathrm{dBm}$ at 


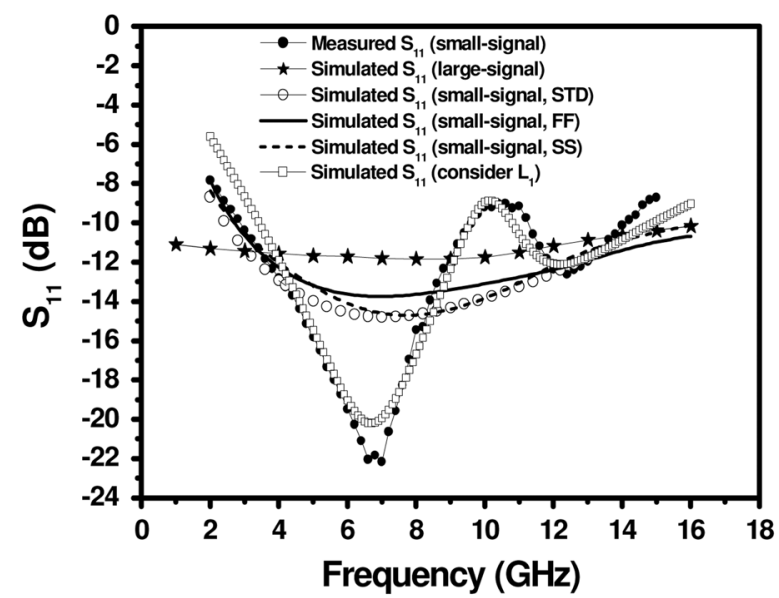

(a)

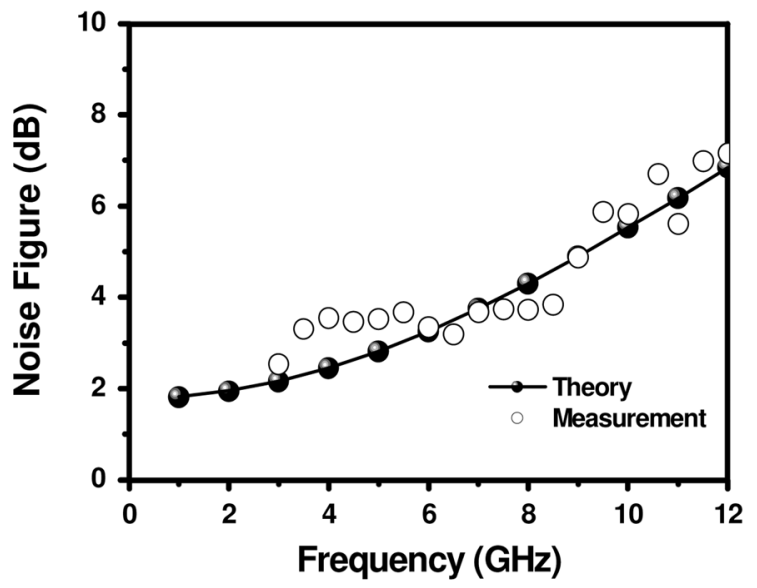

(c)

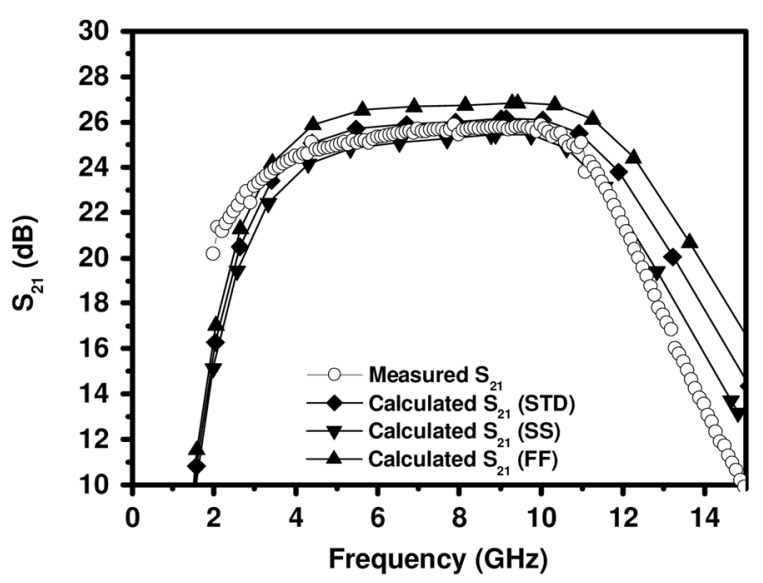

(b)

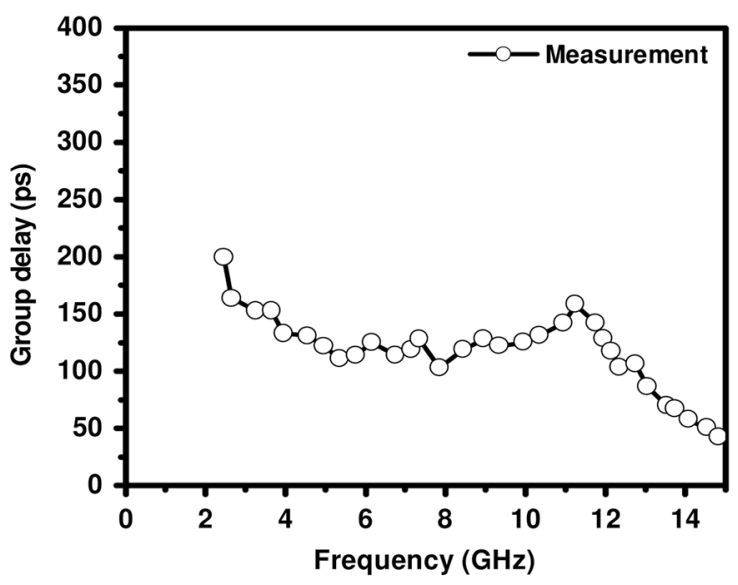

(d)

Fig. 14. (a) Measured $S_{11}$ and simulated $S_{11}$ under process variation and small- and large-signal condition. (b) Measured $S_{21}$ and calculated $S_{21}$ under process variation. (c) Measured and calculated NF [by (20)]. (d) Measured group delay of the SiGe UWB LNA.

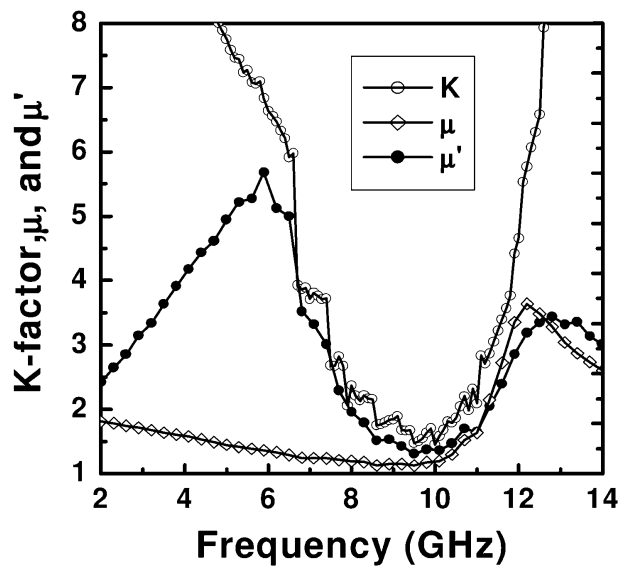

Fig. 15. Measured $K$-factor, $\mu$, and $\mu^{\prime}$ versus frequency characteristics of the SiGe UWB LNA.

3, 5.4, and $10 \mathrm{GHz}$, respectively) simulated by Agilent's Advanced Design System (ADS). In addition, the LNA achieves $P_{1 \mathrm{~dB}}$ of $-27,-25.5$, and $-26 \mathrm{dBm}$ at $3,5.4$, and $10 \mathrm{GHz}$, respectively, which conform well with the calculated results

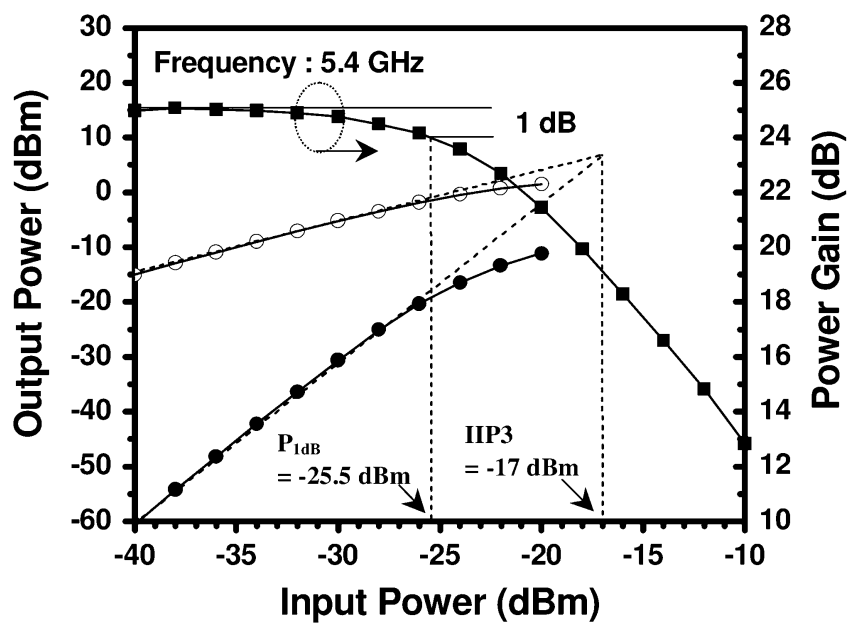

Fig. 16. Measured $P_{1 \mathrm{~dB}}$ and IIP3 of the SiGe UWB LNA.

$(-27.9,-25.7$, and $-25.3 \mathrm{dBm}$ at $3,5.4$, and $10 \mathrm{GHz}$, respectively) [20], and close to those $(-32,-30.5$, and $-27 \mathrm{dBm}$ at 3, 5.4, and $10 \mathrm{GHz}$, respectively) simulated by Agilent's ADS. The UWB IIP3 and $P_{1 \mathrm{~dB}}$ characteristics of the UWB 
TABLE I

SuMMARY OF THE IMPLEMENTED SiGe UWB LNA, AND THE RECENTLY REPORTED STATE-OF-THE-ART SiGe AND CMOS UWB LNA

\begin{tabular}{|c|c|c|c|c|c|c|c|c|c|c|}
\hline & Tech. & $\underset{\mathrm{V}_{\mathrm{DD}}(\mathrm{V})}{\operatorname{Maximum}}$ & $\begin{array}{l}\text { Input matching } \\
\mathrm{BW}(\mathrm{GHz})\end{array}$ & $\begin{array}{c}\text { Peak } S_{21} \\
(\mathrm{~dB})\end{array}$ & $\mid \begin{array}{c}3-\mathrm{dB} \text { BW of } \\
\mathrm{S}_{21}(\mathrm{GHz})\end{array}$ & $\begin{array}{c}\text { Minimum } \\
\mathrm{NF}(\mathrm{dB})\end{array}$ & $\begin{array}{l}\text { Max. Group delay } \\
\text { variation }(\mathrm{ps})\end{array}$ & $\mathrm{P}_{1 \mathrm{~dB}}(\mathrm{dBm})$ & $\begin{array}{c}\text { IIP3 } \\
(\mathrm{dBm})\end{array}$ & $\begin{array}{c}\text { Power } \\
\text { consumption } \\
(\mathrm{mW})\end{array}$ \\
\hline $\begin{array}{l}\text { This } \\
\text { work }\end{array}$ & $\begin{array}{c}0.35 \mu \mathrm{m} \\
\mathrm{SiGe}\end{array}$ & 1.7 & $3-14$ & 26.1 & $3-11.6$ & 2.5 & \pm 28 & $-26^{*}$ & $-17 *$ & 26 \\
\hline [1] & $\begin{array}{c}0.18 \mu \mathrm{m} \\
\mathrm{SiGe}\end{array}$ & 3 & $2.2-8$ & 21 & $3-10$ & 2.5 & NA & $-14.7^{*}$ & $-1 *$ & 30 \\
\hline [2] & $\begin{array}{c}0.18 \mu \mathrm{m} \\
\mathrm{CMOS}\end{array}$ & 1.8 & $2.6-11.7$ & 9.3 & $2.3-9.2$ & 4 & \pm 40 & $-15^{* *}$ & $-6.7 * *$ & 9 \\
\hline [4] & $\begin{array}{c}0.25 \mu \mathrm{m} \\
\mathrm{SiGe}\end{array}$ & 1 & $4-6$ & 10 & 3.4-6.9 & 4.5 & \pm 30 & $-10^{*}$ & NA & 3.5 \\
\hline [5] & $\begin{array}{c}0.18 \mu \mathrm{m} \\
\mathrm{SiGe}\end{array}$ & 3.3 & $\begin{array}{c}0.1-13.6 \\
(<-7.2 \mathrm{~dB})\end{array}$ & 20.3 & $0.1-13.6$ & 1.8 & NA & $-12.5^{* *}$ & $2.1 * *$ & 26 \\
\hline [6] & $\begin{array}{c}0.13 \mu \mathrm{m} \\
\mathrm{CMOS}\end{array}$ & 1.2 & $2.2-10.6$ & 16.5 & $3.1-10.6$ & 2.07 & \pm 27.5 & NA & $-5.1^{+}$ & 9 \\
\hline [7] & $\begin{array}{c}0.25 \mu \mathrm{m} \\
\mathrm{SiGe}\end{array}$ & 2.7 & $3.1-10.6$ & 21 & $3.1-10.6$ & 2.8 & NA & $-19.5^{\#}$ & $-8^{\#}$ & 29.7 \\
\hline [8]-[9] & $\begin{array}{c}0.18 \mu \mathrm{m} \\
\mathrm{CMOS}\end{array}$ & 1.8 & $2.8-10.8$ & 8.5 & $1.3-10.7$ & 4.4 & NA & NA & $8.3^{++}$ & 4.5 \\
\hline$[10]$ & $\begin{array}{c}0.18 \mu \mathrm{m} \\
\mathrm{SiGe}\end{array}$ & 2.5 & $\begin{array}{c}3-10 \\
(<-8.1 \mathrm{~dB})\end{array}$ & 20.8 & $3-10$ & 3.05 & NA & NA & $-11.7^{\#}$ & 42.5 \\
\hline [11] & $\begin{array}{c}0.18 \mu \mathrm{m} \\
\mathrm{CMOS}\end{array}$ & 1.8 & $\begin{array}{c}3.1-10.6 \\
(<-9 \mathrm{~dB})\end{array}$ & 17.5 & $3.1-10.6$ & 3.1 & \pm 48 & NA & NA & 33.2 \\
\hline
\end{tabular}

$\#: 3 \mathrm{GHz} \quad *: 5 \mathrm{GHz}$ **: $6 \mathrm{GHz} \quad$ +: $8 \mathrm{GHz} \quad++: 10 \mathrm{GHz}$

LNA are attributed to the last stage of the LNA, which exhibits UWB characteristics, as explained in Section III. In addition, the low or mediocre IIP3 and $P_{1 \mathrm{~dB}}$ performances of the SiGe HBT UWB LNA are mainly due to its high gain of $25 \mathrm{~dB}$ [see (29)]; i.e., the IIP3 and $P_{1 \mathrm{~dB}}$ performances of the LNA can be improved at the expense of its gain. Fig. 16 shows the measured IIP3 and $P_{1 \mathrm{~dB}}$ characteristics of the SiGe HBT UWB LNA at $5.4 \mathrm{GHz}$.

Table I is a summary of this study along with other reported state-of-the-art UWB amplifiers. Based on Table I, it is found that UWB LNAs with gain higher than $20 \mathrm{~dB}$ ([1], [5], [7], [10], and this study) consume more power (30 $\mathrm{mW}$ in [1], $26 \mathrm{~mW}$ in [5], $29.7 \mathrm{~mW}$ in [7], $42.5 \mathrm{~mW}$ in [10], and $26 \mathrm{~mW}$ in this study) than those with gain near $10 \mathrm{~dB}$. Clearly, if a more advanced SiGe HBT technology is adopted and a lower $S_{21}$ is specified, such as those in [4], the power consumption of our proposed LNA is expected to be largely reduced. In addition, the overall performances of our proposed LNA are expected to be compatible with those in [5] and [6] if a more advanced $0.18-\mu \mathrm{m} \mathrm{SiGe}$ HBT (or $0.13-\mu \mathrm{m}$ CMOS) technology is adopted.

\section{CONCLUSION}

A new methodology of UWB LNA design, which takes advantage of the Miller effect to achieve UWB input impedance matching and the inductive shunt-shunt feedback technique to achieve flat and high UWB $S_{21}$, has been proposed. Based on the proposed methodology, an SiGe UWB LNA was designed and implemented. Very good input matching, power gain, $\mathrm{NF}$, and phase linearity performances were achieved for the
SiGe UWB LNA. These results demonstrate that the proposed methodology is very suitable for UWB LNA design.

\section{ACKNOWLEDGMENT}

The authors are very grateful for the support of the National Chip Implementation Center (CIC), Hsinchu, Taiwan, R.O.C., for chip fabrication, and to Dr. G. W. Huang, National Nano Device Laboratory (NDL), Hsinchu, Taiwan, R.O.C., for high-frequency measurements. Helpful discussions with Prof. H. W. Chiu, National Taipei University of Technology, Taipei, Taiwan, R.O.C., are also appreciated.

\section{REFERENCES}

[1] A. Ismail and A. A. Abidi, "A 3-10-GHz low-noise amplifier with wideband $L C$-ladder matching network," IEEE J. Solid-State Circuits, vol. 39, no. 12 , pp. 2269-2277, Dec. 2004.

[2] A. Bevilacqua and A. M. Niknejad, "An ultrawideband CMOS lownoise amplifier for 3.1-10.6 GHz wireless receivers," IEEE J. SolidState Circuits, vol. 39, no. 12, pp. 2259-2268, Dec. 2004.

[3] Y. Park, C. H. Lee, J. D. Cressler, and J. Laskar, "Theoretical analysis of a low dispersion SiGe LNA for ultra-wideband applications," IEEE Microw. Wireless Compon. Lett., vol. 16, no. 9, pp. 517-519, Sep. 2006.

[4] D. Barras, F. Ellinger, H. Jackel, and W. Hirt, "A low supply voltage SiGe LNA for ultra-wideband frontends," IEEE Microw. Wireless Compon. Lett., vol. 14, no. 10, pp. 469-471, Oct. 2004.

[5] Y. Lu, R. Krithivasan, W. M. L. Kuo, and J. D. Cressler, "A 1.8-3.1 dB noise figure (3-10 GHz) SiGe HBT LNA for UWB applications," in IEEE Radio Freq. Integrated Circuits Symp., Jun. 2006, pp. 45-48.

[6] M. T. Reiha, J. R. Long, and J. J. Pekarik, "A 1.2 V Reactive-feedback 3.1-10.6 GHz ultrawideband low-noise amplifier in $0.13 \mu \mathrm{m}$ CMOS," in IEEE Radio Freq. Integrated Circuits Symp., Jun. 2006, pp. 41-44.

[7] B. Shi and M. Y. W. Chia, "A SiGe Low-Noise amplifier for 3.1-10.6 $\mathrm{GHz}$ ultra-wideband wireless receivers," in IEEE Radio Freq. Integrated Circuits Symp., Jun. 2006, pp. 57-60. 
[8] S. Shekhar, X. Li, and D. J. Allstot, "A CMOS 3.1-10.6 GHz UWB LNA employing stagger-compensated series peaking," in IEEE Radio Freq. Integrated Circuits Symp., Jun. 2006, pp. 49-52.

[9] S. Shekhar, J. S. Walling, and D. J. Allstot, "Bandwidth extension techniques for CMOS amplifiers," IEEE J. Solid-State Circuits, vol. 41, no. 11, pp. 2424-2439, Nov. 2006.

[10] J. Lee and J. D. Cressler, "Analysis and design of an ultra-wideband low-noise amplifier using resistive feedback in SiGe HBT technology," IEEE Trans. Microw. Theory Tech., vol. 54, no. 3, pp. 1262-1268, Mar. 2006.

[11] Y. Lu, K. S. Yeo, A. Cabuk, J. Ma, M. A. Do, and Z. Lu, "A novel CMOS low-noise amplifier design for 3.1-to-10.6-GHz ultra-wideband wireless receiver," IEEE Trans. Circuits Syst. I, Reg. Papers, vol. 53, no. 8, pp. 1683-1692, Aug. 2006.

[12] C. J. Madden, R. L. Van, T. V. Le, and L. D. Nguyen, "A 17 dB gain, 0.1-70 GHz InP HEMT amplifier IC," Int. Solid-State Circuits Tech. Dig., pp. 178-179, Feb. 1994.

[13] Y. Soliman, L. MacEachern, and L. Roy, "A CMOS ultra-wideband LNA utilizing a frequency-controlled feedback technique," in IEEE Int. Ultra-Wideband Conf., Sep. 2005, pp. 530-535.

[14] T. Masuda, T. Nakamura, M. Tanabe, N. Shiramizu, S. I. Wada, T. Hashimoto, and K. Wshio, "SiGe HBT based 24-GHz LNA and VCO for short-range ultra-wideband radar systems," in Int. Solid-State Circuits Conf. Tech. Dig., Nov. 2005, pp. 425-428.

[15] A. S. Sedra and K. C. Smith, Microelectronic Circuits, 5th ed. New York: Oxford Univ. Press, 2004, pp. 840, 1103-1105.

[16] T. Wang, H. C. Chen, H. W. Chiu, Y. S. Lin, G. W. Huang, and S. S. Lu, "Micromachined CMOS LNA and VCO by CMOS-compatible ICP deep trench technology," IEEE Trans. Microw. Theory Tech., vol. 54, no. 2, pp. 580-588, Feb. 2006.

[17] H. W. Chiu, S. S. Lu, and Y. S. Lin, "A $2.17 \mathrm{~dB}$ NF, $5 \mathrm{GHz}$ band monolithic CMOS LNA with $10 \mathrm{~mW}$ DC power consumption," IEEE Trans. Microw. Theory Tech., vol. 53, no. 3, pp. 813-824, Mar. 2005.

[18] Y. T. Lin and S.-S. Lu, "A 2.4/3.5/4.9/5.2/5.7-GHz concurrent multiband low noise amplifier using InGaP/GaAs HBT technology," IEEE Microw. Wireless Compon. Lett., vol. 14, no. 10, pp. 463-465, Oct. 2004.

[19] P. W. Lee, H. W. Chiu, T. L. Hsieh, C. H. Shen, G. W. Huang, and S. S. Lu, "A SiGe low noise amplifier for 2.4/5.2/5.7 GHz WLAN applications," in Int. Solid-State Circuits Conf. Tech. Dig., San Francisco, CA, Feb. 2003, pp. 364-465.

[20] B. Razavi, $R F$ Microelectronics. Englewood Cliffs, NJ: Prentice-Hall, 1998, pp. 22-24, 50-52, 169, 173, 213

[21] H. T. Friis, "Noise figure of radio receivers," Proc. IRE, vol. 32, no. 7, pp. 419-422, Jul. 1944.

[22] D. J. Cassan and J. R. Long, "A 1-V transformer-feedback low-noise amplifier for 5-GHz wireless LAN in 0.18- $\mu \mathrm{m}$ CMOS," IEEE J. SolidState Circuits, vol. 38, no. 3, pp. 427-435, Mar. 2003.

[23] D. K. Shaeffer and T. H. Lee, "A 1.5-V 1.5-GHz CMOS low noise amplifier," IEEE J. Solid-State Circuits, vol. 32, no. 5, pp. 745-759, May 1997.

[24] P. C. H. Lee, J. D. Cressler, and J. Laskar, "The analysis of UWB SiGe HBT LNA for its noise, linearity, and minimum group delay variation," IEEE Trans. Microw. Theory Tech., vol. 54, no. 4, pp. 1687-1697, Apr. 2006.

[25] M. L. Edwards and J. H. Sinsky, "A new criterion for linear 2-port stability using a single geometrically derived parameter," IEEE Trans. Microw. Theory Tech., vol. 40, no. 12, pp. 2303-2311, Dec. 1992.

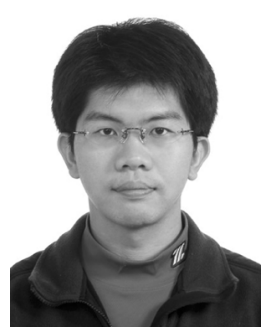

Yu-Tso Lin was born in Hsinchu, Taiwan, R.O.C., in 1978. He received the B.S. degree from Tatung University, Taipei, Taiwan, R.O.C., in 2001, the M.S. degree in electronics engineering from National Taiwan University, Taipei, Taiwan, R.O.C., in 2003, and is currently working toward the Ph.D. degree at National Taiwan University. His master thesis was related to frequency divider and LNA design.

His current research concerns the design of wireless receiver for UWB communication and wireless sensor networks.

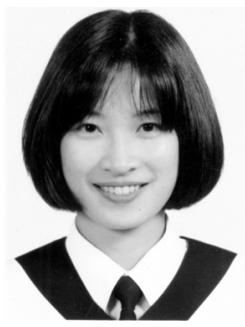

Hsiao-Chin Chen was born in Taipei, Taiwan, R.O.C., on October 6, 1976. She received the B.S. and M.S. degrees in electrical engineering from National Taiwan University, Taipei, Taiwan, R.O.C. in 1998 and 2000, respectively, and is currently working toward the Ph.D. degree at National Taiwan University.

From 2000 to 2003, she was an RF IC Design Engineer with the Airoha Technology Corporation. Her major research interests are RFIC and low-power transmitter design for wireless local area sensor

networks.

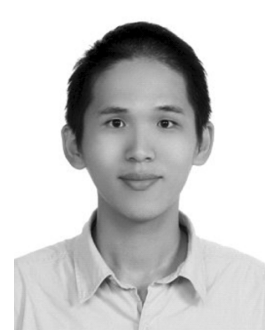

Tao Wang was born in Taipei, Taiwan, R.O.C., in 1980. He received the B.S. degree in electronics engineering from Chang Gung University, Taoyuan, Taiwan, R.O.C., in 2002, the M.S. degree in electronics engineering from National Taiwan University, Taipei, Taiwan, R.O.C., in 2004, and is currently working toward the Ph.D. degree in electronics engineering at National Taiwan University.

His research interests are in the areas of RFICs and microelectromechanical systems (MEMS).

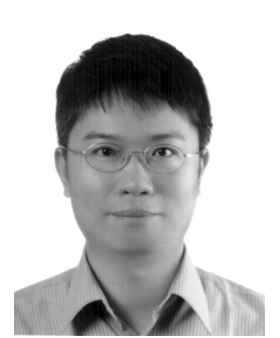

Yo-Sheng Lin (M'02-SM'06) was born in Puli, Taiwan, R.O.C., on October 10, 1969. He received the Ph.D. degree in electrical engineering from National Taiwan, University, Taipei, Taiwan, R.O.C., in 1997. His doctoral dissertation concerned the fabrication and study of GaInP-InGaAs-GaAs doped-channel field-effect-transistors and their applications to monolithic microwave integrated circuits (MMICs).

In 1997, he joined the Taiwan Semiconductor Manufacturing Company (TSMC), as a Principle Engineer for $0.35 / 0.32-\mu \mathrm{m}$ dynamic RAM (DRAM) and $0.25-\mu \mathrm{m} \mathrm{em}$ bedded DRAM technology development with the Integration Department of Fab-IV. Beginning in 2000, he was responsible for $0.18 / 0.15 / 0.13-\mu \mathrm{m}$ CMOS low-power device technology development with the Department of Device Technology and Modeling, Research and Development, and became a Technical Manager in 2001. In August 2001, he joined the Department of Electrical Engineering, National Chi Nan University (NCNU), Taiwan, R.O.C., where he is currently a Professor. From June to September 2004, he was a Visiting Researcher with the High-Speed Electronics Research Department, Bell Laboratories, Lucent Technologies, Murray Hill, NJ. He is currently a Visiting Professor with the Department of Electrical Engineering, Stanford University, Stanford, CA (February 2007-January 2008). His current research interests are in the areas of characterization and modeling of RF active and passive devices (especially 30-100-GHz interconnections, inductors, and transformers for millimeter-wave (Bi)CMOS ICs), and RFICs/monolithic microwave integrated circuits (MMICs).

Dr. Lin was a recipient of the Excellent Research Award presented by the NCNU in 2006.

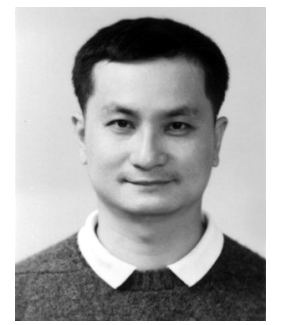

Shey-Shi Lu (''89-M'91-SM'99) was born in Taipei, Taiwan, R.O.C., on October 12, 1962. He received the B.S. degree from National Taiwan University, Taipei, Taiwan, R.O.C., in 1985, the M.S. degree from Cornell University, Ithaca, NY, in 1988, and the Ph.D. degree from the University of Minnesota at Minneapolis-St. Paul, in 1991, all in electrical engineering. His M.S. thesis concerned the planar doped barrier hot electron transistor. His doctoral dissertation concerned the uniaxial stress effect on $\mathrm{AlGaAs} / \mathrm{GaAs}$ quantum well/barrier structures.

In August 1991, he joined the Department of Electrical Engineering, National Taiwan University, where he is currently a Professor. Since August 2007, he has also been the Director of the Graduate Institute of Electronics Engineering, National Taiwan University. His current research interests are in the areas of RFIC/MMICs and micromachined RF components. 